\title{
Contrast-enhanced low mechanical index endoscopic ultrasound (CELMI-EUS)
}

This report describes contrast-enhanced low mechanical index endoscopic ultrasound (CELMI-EUS), a new real-time technique with high resolution.

A 73-year-old man presented with unspecific abdominal pain and suspected liver cirrhosis ( $\odot$ Fig. 1) and a pancreatic mass. CELMI-EUS was performed using the Hitachi 900 HV device combined with an electronic echo endoscope (Hitachi/Pentax EG3870UTK; Hitachi, Tokyo, Japan) and adapted dynamic contrast harmonic wideband pulsed inversion software, with low mechanical index (0.090.30 ), before and up to 180 seconds after injection of $4.8 \mathrm{ml}$ SonoVue (Bracco, Milan, Italy). To improve the contrast in microvessels, microbubble tracing imaging (MTI) was applied.

Enhancement of the liver parenchyma persisted for up to 5 minutes under continuous scanning conditions. No significant destruction of bubbles was visualized. CELMI-EUS revealed arterial rim enhancement and delineated central necrosis, typical signs of neuroendocrine metastases ( $\bullet$ Fig. 2 and $\bullet$ Video 1 ). Histological examination revealed neuroendocrine tumor metastasis.

While EUS has become established over the past 20 years, real-time contrast applications using low mechanical index were restricted to the transabdominal approach [2]. Recent advances in technology have supported the development of new endoscopic systems, making it possible to use CELMI-EUS as with a recently described prototype [3]. To distinguish between contrast medium signals and tissue signals, two ultrasound pulses with a $180^{\circ}$ inverted phase are sent out at the same vector line. The received echo information from these two pulses is added, eliminating the linear information of the fundamental (tissue) signals. The nonlinear echo information of the contrast media can be displayed

\section{Video 1}

Contrast-enhanced endoscopic ultrasound demonstrated peripheral rim enhancement of a liver lesion, which was shown histologically to be a metastasis of a neuroendocrine tumor of the pancreas. (See also $\odot$ Fig. 2.)

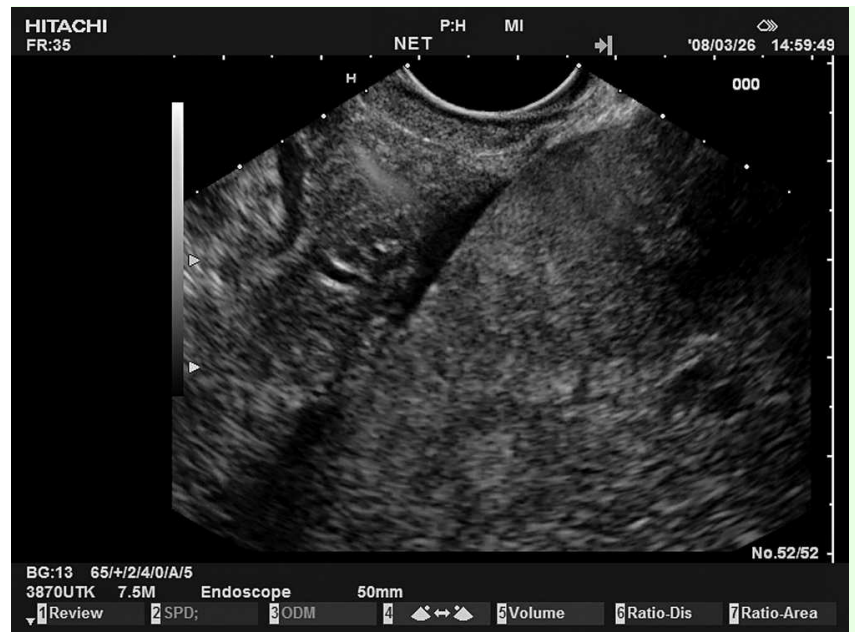

Fig. 1 Conventional B-mode imaging showed diffuse liver disease and ascites.

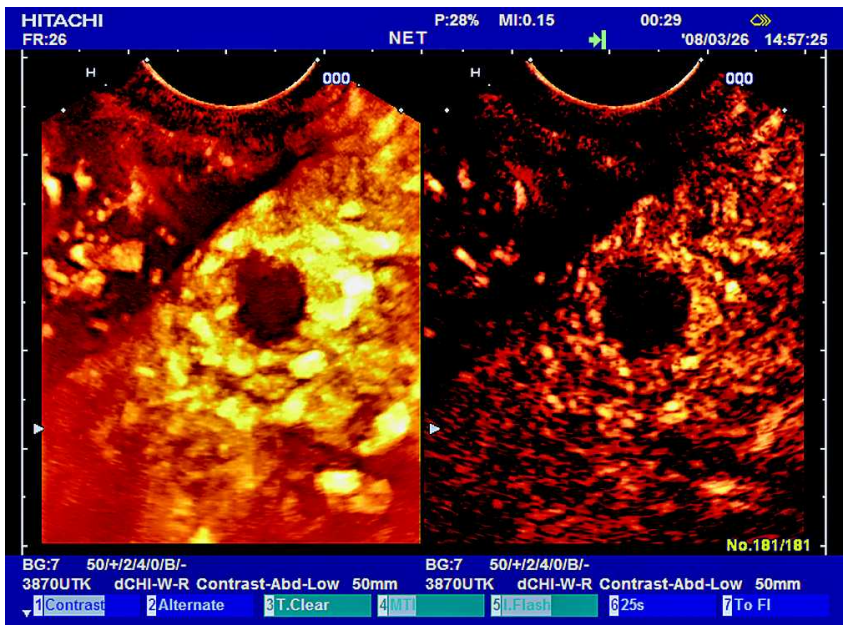

Fig. 2 Contrastenhanced endoscopic ultrasound demonstrated peripheral rim enhancement of a liver lesion, which was shown histologically to be a metastasis of a neuroendocrine tumor of the pancreas. (See also $\odot$ Video 1.)

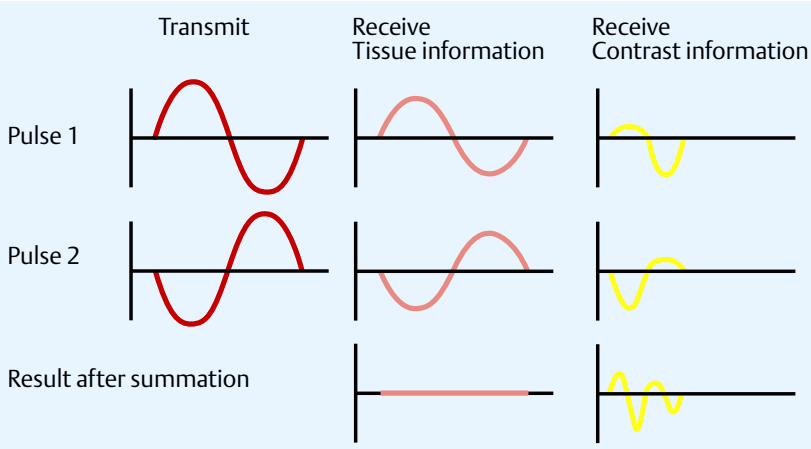

Fig. 3 To distinguish between contrast medium signals and tissue signals, two ultrasound pulses with a $180^{\circ}$ inverted phase are sent out at the same vector line.

The received echo information from these two pulses is summed and the nonlinear echo information from the contrast medium is displayed.

(๑ Fig. 3) [3]. CELMI-EUS may improve our understanding of morphologic imaging methods through additional analysis of functional criteria such as perfusion, with better characterization of, for exam- ple, tumors, lymph node staging, and organ infiltration. This improved image documentation will enhance confidence in the method. 
Endoscopy_UCTN_Code_CCL_1AF_2AG_3AD

\section{F. Dietrich}

Medical and Imaging Department, CaritasKrankenhaus, Bad Mergentheim, Germany

\section{References}

1 Mörk H, Ignee A, Schuessler G et al. Analysis of neuroendocrine tumour metastases in the liver using contrast enhanced ultrasonography. Scand J Gastroenterol 2007; 42: 652-662

2 Claudon $M$, Cosgrove $D$, Albrecht $T$ et al. Guidelines and good clinical practice recommendations for contrast enhanced ultrasound (CEUS) - Update 2008. Ultraschall Med 2008; 29: $28-44$

3 Dietrich $C F$, Ignee A, Frey $H$. Contrast enhanced endoscopic ultrasound with low mechanical index, a new technique. Z Gastroenterol 2005; 43: 1219-1223
Bibliography

DoI 10.1055/s-0028-1119491

Endoscopy 2009; 41: E43-E44

(c) Georg Thieme Verlag KG Stuttgart · New York . ISSN 0013-726X

\section{Corresponding author}

\section{F. Dietrich, MD}

Innere Medizin 2

Caritaskrankenhaus Bad Mergentheim

Uhlandstr. 7

D-97980 Bad Mergentheim

Germany

Fax: +49-7931-58-2290

christoph.dietrich@ckbm.de 